\title{
Coulisses
}

Revue de théâtre

\section{Les didascalies dans le théâtre de J. Giraudoux}

\section{Françoise Bombard}

\section{CpenEdition}

Journals

Édition électronique

URL : https://journals.openedition.org/coulisses/983

DOI : $10.4000 /$ coulisses. 983

ISSN : 2546-9460

\section{Éditeur}

Presses universitaires de Franche-Comté

\section{Édition imprimée}

Date de publication : 31 décembre 2009

Pagination : 101-118

ISBN : 978-2-84867-270-0

ISSN : $1150-594 \mathrm{X}$

\section{Référence électronique}

Françoise Bombard, "Les didascalies dans le théâtre de J. Giraudoux », Coulisses [En ligne], 39 | Automne 2009, mis en ligne le 30 novembre 2016, consulté le 29 décembre 2022. URL : http:// journals.openedition.org/coulisses/983; DOI : https://doi.org/10.4000/coulisses.983

Ce document a été généré automatiquement le 29 décembre 2022.

Tous droits réservés 


\title{
Les didascalies dans le théâtre de J. Giraudoux
}

\author{
Françoise Bombard
}

1 Une idée fort répandue est que le théâtre de J. Giraudoux est pauvre en didascalies ${ }^{1}$. S'il est vrai qu'il offre peu de didascalies descriptives, en revanche, nombreuses sont les didascalies narratives. Les modes d'écriture didascalique doivent être mis en relation avec les choix esthétiques et dramaturgiques de Giraudoux : qu'en est-il de la mimesis, de la déréalisation, de la théâtralisation? Certaines didascalies s'adressent de toute évidence au lecteur et posent le problème de choix de mise en scène - les ignorer ou non -, qu'il s'agisse d'humour obtenu par le tissage des didascalies et des répliques ou de l'intrusion du «non visible ».

2 Rappelons, après A. Helbo, que le destinataire des didascalies est « le praticien (ou le lecteur, praticien virtuel) » : il s'agit de « lui enjoindre de construire tel ou tel ensemble de conditions d'énonciation spatiale » ainsi que le «mode d'investissement de l'espace par les personnages (occupation du lieu, mouvement, gestuelle) $»^{2}$. Selon M. Ezquerro, « [...].le texte didascalique n'est pas destiné seulement à la lecture du metteur en scène, mais à celle d'un nombre croissant de lecteurs "non-professionnels»", et il a pour fonction de "faire surgir dans l'imagination de son lecteur des images précises et complexes qui ne sont pas inévitablement induites par ce que disent les personnages. $»^{3}$.

\section{Le prélude didascalique}

3 À l'instar de la plupart de ses contemporains et contrairement à la pratique courante $\mathrm{du}$ théâtre classique, Giraudoux ne rédige aucune macrodidascalie après la liste des personnages pour préciser le lieu de l'action ou le moment, exception faite, évidemment, des pièces en un acte ${ }^{4}$. Les didascalies liminaires de chaque acte remplissent donc pour les autres pièces la même fonction pragmatique pour le metteur en scène et il s'agira pour le lecteur de construire ce que J.-P. Ryngaert appelle «la scène imaginaire $»^{5}$. 
4 Nous avons identifié trois pratiques d'écriture des ces débuts d'actes : des didascalies réduites au lieu de l'action, renouant avec la pratique du théâtre classique français, ceci pour Judith, les actes I et II d'Intermezzo, Ondine, l'acte I de La Folle de Chaillot, les actes I et II de Pour Lucrèce; des didascalies à proprement parler descriptives comportant la mention d'un ou de plusieurs objets d'un décor dans Siegfried, Amphitryon 38, Tessa, La Guerre de Troie n'aura pas lieu, Electre et les actes II de La Folle de Chaillot et III de Pour Lucrèce ; enfin, l'absence de didascalie liminaire dans Sodome et Gomorrhe $e^{6}$.

5 Dans les pièces "antiques", les didascalies structurent un espace inspiré de l'Iliade comme de la tragédie grecque :

Une terrasse près d'un palais (Amph., I, p. 115),

Terrasse d'un rempart dominé par une terrasse et dominant d'autres remparts. (GT, I,

p. 483),

Cour intérieure dans le palais d'Agamemnon. (El., I, p. 597).

6 La sobriété de ces indications a pour corollaire l'élaboration progressive de l'architecture de ces palais par d'autres didascalies en cours d'actes, voire sa déréalisation par le dialogue (par exemple celui des Petites Euménides et du Jardinier au début d'Electre). En revanche, la didascalie liminaire du second acte de La Guerre de Troie n'aura pas lieu est véritablement descriptive :

Square clos de palais. À chaque angle, échappée sur la mer. Au centre, un monument, les portes de la guerre. Elles sont grandes ouvertes. (GT, II, p. 512).

$7 \quad$ L'enjeu du débat qui a opposé les personnages au premier acte est matérialisé au centre du plateau par un objet pris au temple de Janus à Rome $^{7}$ et la découverte sur le paysage maritime qui semble une ouverture dans ce lieu clos prépare en fait l'arrivée des Grecs et donc la tragédie.

Ondine propose des décors de conte :

Cabane de pêcheur. (Ond., I, p. 761),

Salle d'honneur dans le palais du roi. (Ond., II, p. 790),

Cour du château. (Ond., III, p. 826).

9 Cet « espace vide » est rempli au premier acte par le dialogue des objets nécessaires aux marques de l'hospitalité accordées au Chevalier, tandis qu'au second acte, «colonnes » et "escalier» donnent un semblant de réalité au palais dans lequel fonctionnent pourtant... des « jets d'eau » (Ond., II, p. 825).

10 À rapprocher bien sûr dans trois pièces modernes les lieux de la sociabilité qui perdent vite leur caractère euphorique :

Belle terrasse de café de luxe. Au Bois ou sur la Seine. (C., p. 727),

Terrasse chez Francis, place de l'Alma. (FC, I, p. 951),

À Aix-en-Provence. Vers 1868. C'est l'été. Terrasse d'une pâtisserie sous les platanes. (Luc., I, p. 1035).

11 Le café Francis était fréquenté par Giraudoux, Jouvet et ses comédiens au sortir de la Comédie des Champs Elysées. Pour Lucrèce, la dernière pièce de Giraudoux, par une double stipulation temporelle rarissime chez lui inscrit la précision référentielle dans une couleur locale non plus parisienne mais aixoise.

12 Le cas le plus étonnant est celui des indications temporelles d'une souveraine imprécision dans Intermezzo: "Vers le soir» (Int., I, p. 279), "Crépuscule encore lointain » (Int., II, p, 306). Le Spectre préfère ces moments incertains pour ses entrevues avec Isabelle. Pourtant, le décor paraît fort innocent dans les formulations didascaliques : 
La campagne. Une belle prairie. (Int., I, p. 279)

Un autre aspect de la campagne. Bosquets de hêtres. (Int., II, p. 306).

13 À l'inverse, la didascalie liminaire du troisième acte, d'une exceptionnelle précision, pose des problèmes pour la réalisation, le Spectre devant pénétrer dans cet espace $\operatorname{clos}^{8}$ :

La chambre d'Isabelle. Un balcon à deux fenêtres d'où l'on voit la place de la petite ville, sur laquelle donne aussi une porte fermée. [...] (Int., III, p. 333).

Il apparaît donc que dans le prélude didascalique, les macro ou micro didascalies prescriptives sont rarement descriptives, le plus souvent lacunaires.

\section{Le « postlude didascalique »}

Selon S. Golopentia,

Le postlude didascalique inclut le plus souvent l'un des deux sous-ensembles de macrodidascalies qui suivent (ou les deux ensemble) : (a) les macrodidascalies de la clôture du spectacle et (b) les macrodidascalies de la clôture textuelle. ${ }^{9}$

L'accent est mis de façon très nette sur la théâtralité dans L'Impromptu de Paris où le jeu sur un élément de la machinerie, la " gloire », accompagne la clôture textuelle :

La gloire, au lieu de descendre, se met à remonter.

Jouvet: Qu'est-ce que tu fiches, Léon, tu m'as compris?

Léon : Le mouvement s'est détraqué. Je n'ai plus de commandes!

Robineau: Ne vous affolez pas, Messieurs. Quelle que soit l'issue par où je sors de

cette scène, l'Etat connaîtra vos désirs !

La Petite Véra, criant : Restez droit, calme.

Robineau : Je reste droit, calme...

Raymone : Voilà qu'il monte au ciel !

Robineau, montant : Tant mieux !... C'est du théâtre ! Il disparaît. Rideau. (IP, 4, p. 724).

La clôture du spectacle, légèrement différée par les caprices de la "gloire ", permet à la clôture textuelle de se faire sur deux plans, à savoir celui du départ du député, clôture d'une fable qui le place à l'origine dans la position du fâcheux qui vient perturber une répétition, et celui du discours sur les politiques culturelles dans le domaine du théâtre qui est, lui, d'une brûlante actualité et renvoie aux préoccupations de Giraudoux autant qu'à celles de Jouvet metteur en scène du Cartel. En outre, cette clôture parodie les interventions de deus ex machina, ce que souligne plaisamment la réplique de Raymone. Dès lors, le mot «rideau », qui indique de manière conventionnelle la fin, reprend sa dimension théâtrale.

La fin de La Guerre de Troie n'aura pas lieu présente une écriture encore plus complexe du postlude didascalique. À la suite d'une indication sur le jeu d'Hector et d'Andromaque, "(Il essaie de détacher les mains d'Andromaque qui résiste, les yeux fixés sur Demokos.) » et après la réplique triomphante d'Hector, «La guerre n'aura pas lieu, Andromaque!", nous avons une fausse clôture textuelle par un jeu sur les temps verbaux : «Le rideau qui avait commencé à tomber se relève peu à peu. ", signifiant par là l'erreur d'Hector, le véritable dénouement lui échappant complètement. La véritable clôture textuelle vient après les cris de Demokos et le lynchage d'Oiax : «Hector : Elle aura lieu. » (ibid.). Cette réponse au titre et aux illusions pacifistes se matérialise alors par une didascalie :

Les portes de la guerre s'ouvrent lentement. Elles découvrent Hélène qui embrasse Troilus.

(ibid.) 

personnages extravagants. Comme Giraudoux n'est pas coutumier de ce genre de précision didascalique, il est évident que nous avons affaire à ce "réalisme de la fantaisie » qui, à ses yeux, est bien plus intéressant que le réalisme ordinaire ${ }^{13}$. La 
juxtaposition d'éléments de costumes qui semblent pris à plusieurs gravures de mode est une constante dans les indications concernant les quatre « Folles». Pour la Folle de Chaillot, le dernier objet nommé nous ramène au vingtième siècle et à la décadence de l'aristocratie :

Jupe de soie faisant la traîne. [...] Souliers Louis XIII. Chapeau Marie-Antoinette. Un face à main pendu par une chaîne. Un camée. Un cabas. (FC, I, p. 964).

Le costume de Constance, la Folle de Passy, est tout aussi hétéroclite, tandis que celui de Gabrielle, la Folle de Saint-Sulpice, renvoie implicitement au temps des robes à tournure et du théâtre naturaliste :

Constance en robe blanche à volants avec chapeau Marie-Antoinette à voilette violette, solides bottines élastiques. Gabrielle, faussement simple avec toque et manchon 1880 [...]. (FC, II, p. 993).

Défi au temps et au costumier de théâtre que ce costume qui fait un étrange compromis entre la République et le cléricalisme ${ }^{14}$, le mot " concorde " pris au pied de la lettre ne manquant alors pas de sel : bien sûr, il faut y voir également, comme pour les trois autres « folles », un lieu parisien, la place du même nom :

Joséphine, la Folle de la Concorde, entre majestueusement, dans un accoutrement mi-

Fallières, mi-papal. Charlotte blanche. (FC, II, p. 1006).

Ces quatre costumes illustrent le refus du présent chez les quatre Folles, moyen pour elles de nier la vieillesse. Objets désuets et objets modernes nous guident vers une interprétation: la pièce oppose clairement deux moments de l'histoire, un autrefois mythique, temps de l'élégance, de la jeunesse des quatre Folles et un aujourd'hui trivial et décevant : la traîne « est relevée par une pince à linge de métal » $\left(F C\right.$, p. 964) ${ }^{15}$.

Il est un mode d'écriture didascalique proche de la caricature dans La Folle de Chaillot, celui qui concerne les "mecs", ces derniers apparaissant par groupes, et avec des signes distinctifs récurrents, "moustaches" et "cigares", le trait allant jusqu'à l'amplification comique d'un groupe à l'autre dans des séries qui correspondent à ceux qui font leur entrée: phrases nominales brèves, puis phrases réduites à des adjectifs avant la phrase qui amplifie les précédentes et de laquelle l'objet «Cigares » a disparu, ce qui équivaut au trait incisif du caricaturiste :

Moustaches en virgules. Complets prince de Galles. Cigares. (FC, II, p. 1022),

Tenues bigarrées. Cigares. (FC, II, p. 1023),

Barbus. Ventrus. Moustachus. Surtout familiers. Cigares. (FC., II, p. 1024),

Ils sont de plus en plus barbus, moustachus, Surtout familiers. Cigares. (FC., II, p. 1024),

Ils sont de plus en plus barbus, moustachus, familiers, ventrus. (ibid.),

et, pour leurs compagnes: "Tenue nette. Cigarettes.» (FC, II, p. 1026). Ecriture d'un registre clairement satirique : l'uniformisation prônée par les «mecs» comme garant de l'ordre social leur est appliquée, dans un comique de « l'arroseur arrosé $»^{16}$.

D'autres didascalies descriptives sont clairement prescriptives: elles indiquent le comportement d'un personnage de façon relativement conventionnelle. Pour marquer la jalousie de Pâris à l'égard de Troïlus, "Pâris, un peu énervé: Tu cries bien fort, Hélène !» (GT, II, 2, p. 514), ou, après l'exigence du Chevalier, à savoir cuisiner la truite au bleu, "Effroi d'Auguste et d'Eugénie » qu'inquiète la réaction d'Ondine (Ond., I, 2, p. 765).

Très rare, la mention de l'adresse à un personnage mérite d'être signalée : la première fois, elle distingue le destinataire parmi d'autres personnages : «Egisthe, au mendiant : Vous dites, vous? » (El., I, 4, p. 625), la seconde fois, elle met à mal un procédé théâtral 
codifié, l'aparté, de façon désinvolte : « Le mendiant, à lui-même, mais à voix haute. » (El., I, 12, p. 636).

\section{Les didascalies narratives} : "Fracas. Jupiter paraît escorté de Mercure." (Amph., III, 3, p. 183). Nous mentionnerons l'emploi fréquent du verbe « apparaître » pour des personnages dont la présence perturbe le dialogue, celui d'Oreste et d'Electre par exemple : "Apparaît au fond Clytemnestre.»(El., I, 10, p.634). Plus fréquemment, il s'agit d'interventions 
surnaturelles : La Paix (GT, I, 10, p. 511) ${ }^{19}$, Une petite Euménide (El., I, 11, p. 636), « Une tête de vieillard couronnée ", "Une charmante tête de naïade ", Une ondine (Ond., I, 8, p. 762-63 et 782), et jusqu'au Monsieur de Bellac dont nous ne saurons jamais s'il joue à être Apollon ou si Apollon visite la jeune fille ${ }^{20}:$ «La tête du Monsieur de Bellac apparaît et encourage Agnès » (Ap., 3, p. 926). «La Fille de vaisselle apparait. » (Ond., III, 4, p. 843), la didascalie commente d'ailleurs l'ambiguïté du personnage: "Pour les uns la beauté même, pour les autres, une souillon.» (ibid.) $)^{21}$. D'autant plus remarquable est alors l'usage de ce verbe dans La Folle de Chaillot : pour la Folle ( $F C, \mathrm{I}, \mathrm{p} .964)$, pour Irma ( $F C, \mathrm{I}, \mathrm{p} .955)$ et pour les comparses (FC, II, p. 1009), ce qui tend à faire d'eux des personnages hors du commun par un renversement significatif (dans la société, ils sont ou des exclus ou des subalternes, marginaux ou petit peuple de Paris). La «Folle» et Irma deviennent des figures mythiques de cerbère pour l'une et de déesse des enfers pour l'autre à l'acte II, puisqu'elles condamneront les «mecs » à être enterrés vivants dans le souterrain de la rue de Chaillot.

Un jeu d'entrées et de sorties précipitées introduit le comique par l'aspect mécanique qu'amène la répétition des formulations des déplacements du garçon au début de Pour Lucrèce :

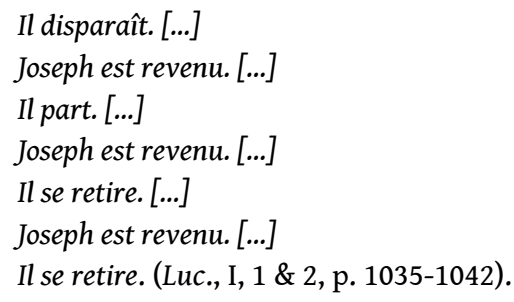

En général, Giraudoux ne se préoccupe guère de la sortie de ses personnages: six sorties sont indiquées dans le théâtre complet. La première relève de la convention pour dire qui reste en scène : "Tous sortent, moins Judith, Joachim, Paul et le garde. [...).» (Jud., III, 5, p. 266). Une autre souligne l'un des ressorts de la tragédie à l'issue d'une scène d'exposition pour le moins bouffonne : "Sortent les Petites Euménides, devant qui s'écartent avec terreur les invités.» (El., I, 1, p. 602). Mais par quatre fois Giraudoux a recours à la formule latine « exit » : il est difficile de ne pas y voir une parodie de l'usage classique lorsqu'il s'agit de personnages de comédie comme la Secrétaire et le Président de l'Office des Grands et petits Inventeurs dans L'Apollon de Bellac : le rapprochement du latin et $\mathrm{du}$ nom ridicule du personnage renforce le comique: "Exit Mademoiselle Chèvredent. " (Ap., 7, p. 936). La formule peut au contraire conférer une dimension tragique à la sortie d'un personnage : "Exit le Procureur. " après que l'entremetteuse a raconté le soi-disant viol de Lucile par Marcellus (Luc., III, 5, p. 111022). Ailleurs, le verbe a une allure prétentieuse comme le personnage qui sort, ce qui ménage un contraste avec les entrées simultanées d'une dame de la cour et d'un pêcheur: "Exit le Chambellan. Entrent d'un côté Violante, de l'autre, Auguste » (Ond., II, 7, p. 801).

\section{Les didascalies narratives gestuelles}

Elles sont de moins en moins fréquentes à mesure qu'on avance dans la production théâtrale de Giraudoux, exception faite de La Folle de Chaillot, exception qui peut s'expliquer par le règne de la fantaisie dans cette œuvre: faut-il voir dans cette évolution chronologique le retour à un certain classicisme? 
41 Pour prescriptives qu'elles soient, les didascalies gestuelles sont essentiellement l'occasion de produire pour le lecteur des effets de décalage qui le dédommagent du comique gestuel qu'introduit à la représentation le jeu des comédiens, un gag par exemple :

D'un mouvement coquet la Folle lance l'écharpe en arrière, renverse le verre du président sur son pantalon et s'en va. (FC, I, p. 965).

Nous voudrions attirer l'attention sur un procédé récurrent chez Giraudoux : il emploie les temps du passé en concurrence avec le présent au lieu de rédiger plusieurs didascalies. Ceci s'avère parfois être un jeu sur le temps. Ainsi, dans Judith, les verbes des didascalies gestuelles sont au passé, le premier au plus-que-parfait, le second au passé composé, tandis que tous les verbes qui désignent les déplacements successifs de Jean, à savoir « disparaître », " revenir », " se précipiter », sont au présent :

Jean, qui avait tiré son épée, disparaît vers la chambre d'Holopherne. [...] Jean revient aussitôt transfiguré. Il se jette aux pieds de Judith. [...] Il se précipite à nouveau vers la chambre, Suzanne s'est jetée aux genoux de Judith. (Jud., III, 2 et 3, p. 258-259).

Ceci suggère le mouvement, c'est-à-dire un élément de nature spatiale, par un élément de nature temporelle: l'inverse de ce qui se passe sur un plateau où l'espace peut exprimer le temps. Mais l'emploi du passé peut donner l'impression d'un ralenti s'il intervient après des didascalies au présent : dans La Guerre de Troie n'aura pas lieu, aux retrouvailles d'Hector et d'Andromaque exprimées par des exclamatives puis par le jeu "(Ils s'étreignent)», succède comme un alanguissement par le rythme ternaire descendant (7/ 7/6) et les pauses marquées par la ponctuation ralentissent le rythme : équivalent littéraire d'un jeu empreint de gravité et de tendresse, Cassandre venant d'annoncer qu'Andromaque attend un enfant :

Il l'a prise dans ses bras, l'a amenée au banc de pierre, s'est assis près d'elle. Court silence.

(GT, I, 3, p. 486).

Ces exemples tendent à prouver que Giraudoux a le souci de rendre perceptible au lecteur, par un artifice d'écriture, ce qui est une donnée immédiate pour un spectateur : l'enchaînement des répliques et du jeu ou leur simultanéité, peut-être encore davantage ce qui relève vraiment de la mise en scène, le rythme des actes et des paroles.

\section{Les sons off}

Les mentions de sons off sont toujours en prise directe sur l'action : la "Voix céleste » dit la vérité légendaire des exploits d'Hercule et des amours de Jupiter et d'Alcmène (Amph., III, 1, 2, 3) que Thèbes va célébrer : «Bruits de la foule et musique.» (Amph., III, 3, p. 180). Au second acte d'Electre, aube et guerre vont de paire : « Un coq. Une trompette lointaine. » (El., II, 1, p. 643). Il en va de même lorsque la tension monte à Troie : «On entend des clameurs du côté du port. » (GT, II, 5, p. 520), « La musique des Grecs éclate. » (p. 526).

Giraudoux peut aussi jouer du « off» sur le mode comique : le "fâcheux », le député Robineau qui vient à l'Athénée voir Jouvet, est d'abord... une succession de bruits ("Bruit de chute.», "Bruit sourd.", "Sons indistincts. ») : le personnage s'empêtre dans les décors remisés en coulisses (IP, 1, p. 693). 


\section{Théâtralisation} précises et nombreuses, phénomène assez rare chez Giraudoux pour qu'il soit remarqué, et s'appuie sur la personnification grâce à l'emploi de verbes d'action pour l'inanimé. Ainsi, après les efforts du Contrôleur pour fermer hermétiquement les issues de la chambre d'Isabelle, «La porte verrouillée s'ouvre. Le Spectre paraît [...]. » (Int., II, 3, p. 345). À chaque dénégation du Chambellan à l'acte II d'Ondine répond le surgissement immédiat des objets les plus inattendus :

Le Chambellan: [...] On ne fait point passer de comètes avec leur queue, on ne fait point monter des eaux la ville d'Ys [...] L'Illusionniste: Si. Une comète passe. La ville d'Ys émerge. Le Chambellan : [...] On ne fait point entrer le cheval de Troie [...], on ne dresse point les Pyramides [...] Le cheval de Troie entre. Les Pyramides se dressent. (Ond., II, 1, p. 792).

51 Tous ces mouvements sont un défi aux lois de la pesanteur d'autant qu'aucune machinerie ne semble présider à ces déplacements puisque les didascalies n'énoncent rien de plus qu'une sorte de réponse ironique par les faits à l'incrédulité du Chambellan.

Un procédé différent est utilisé dans La Folle de Chaillot : à l'acte II, l'Egoutier révèle à la Comtesse le secret du souterrain et une didascalie dissipe pour nous le mystère du mouvement : «Il appuie sur un coin de la plinthe. Un pan de mur pivote. » (FC, II, p. 992). S'il paraît bien peu probable, dans la réalité, que tout un pan de mur puisse, sans risque 
d'effondrement, se mouvoir ainsi, il est possible d'imaginer une explication tenant aux possibilités techniques du plateau, ce que ne confirme aucune didascalie, Giraudoux ayant une fois de plus confié à Jouvet le soin de trouver une solution. Lorsqu'Aurélie applique elle-même le procédé, deux signes tendent à en faire une opération magique, le chiffre trois et le verbe donnant une réponse positive de l'objet à une sollicitation rituelle de nature irrationnelle : «La Folle appuie trois fois sur la plinthe et le pan du mur s'ouvre. » (FC, II, p. 1022).

Ce théâtre comporte en outre quelques didascalies qui laissent perplexe.

À la fin de La Folle de Chaillot, « le fictif est enchâssé dans le scénique ${ }^{26}$ :

À partir de ce moment, les paroles des amis de la Folle ne sont plus perceptibles. Ils parlent entre eux, pleins de joie. On voit leurs lèvres remuer, mais on n'entend que le sourd-muet. Le mur opposé au mur du souterrain s'est ouvert, et des cortèges sortent, que seule la Folle voit... [...] (FC, II, p. 1029).

Pour les comparses de la Folle, la pantomime n'a en soi rien d'inimaginable, mais comment "entendre " un sourd muet à moins d'avoir pris modèle sur Irma qui est capable de «li[re] ses mimiques » ${ }^{27}$, autrement dit de déchiffrer le langage des signes, ce qui, lors d'une représentation, relève de l'exploit pour les spectateurs même si le comédien surjoue à l'avant-scène, ou s'il va parmi les spectateurs? Mieux encore, après le non audible, Giraudoux nous propose du non visible, ce qui est un comble au théâtre : ces "cortèges que seule la Folle voit...", et tous les metteurs en scène de les rendre visibles, tant le scandale est grand de cet onirisme !

À l'inverse, Giraudoux prétend rendre visible l'immatériel: à peine "tué » par les Bourreaux, «identique au corps étendu, un Spectre monte. Tous les assistants l'aperçoivent l'un après l'autre. [...] » (Int., II, 7, p. 330). Et Jouvet de s'interroger, d'interroger l'auteur... qui récidive autrement : à l'issue du procès intenté à Ondine, «Le roi des ondins, d'un geste, a changé le bourreau en statue de neige rouge. » (Ond., III, 5, p. 845). La métamorphose du bourreau, par son excès de matérialité, paraît s'opposer à l'immatérialité du second Spectre d'Intermezzo, or, il n'en est rien : comme n'importe quel bonhomme de neige, la «statue de neige rouge » est promise à l'évanouissement par fonte et par évaporation, surtout sous les feux de la rampe.

Et que dire des anneaux du Jongleur? «Le jongleur termine par des jongleries dans le ciel. Les anneaux ne redescendent pas, mais le chanteur est revenu. » (FC, I, p. 958). Le Jongleur lance-t-il ses anneaux si haut qu'ils disparaissent à la vue ? Est-ce comme au cirque où les anneaux peuvent s'accrocher en haut du chapiteau? Est-ce que l'atmosphère les retient ? "L'air lui-même n'est plus comme autrefois. ", a-t-il confié à la Comtesse (FC, I, p. 985). La construction de la phrase établit en outre une relation pour le moins inattendue entre la perte des anneaux et l'arrivée du Chanteur des rues, comme si des numéros se succédaient, le second faisant oublier le premier. Le prodige trouve donc plusieurs explications, l'une de type écologique et fantaisiste à la fois, l'autre, rationnelle, inspirée des arts du cirque.

Magicien ou régisseur, le Droguiste d'Intermezzo " souffle dans son diapason. La nature s'ordonne d'après sa note et résonne tout entière [...]. » (Int., II, 5, p. 327). À la fin du Prélude de Sodome et Gomorrhe, "L'archange s'éteint. Le jardinier d'éloigne.» (Sod., p. 859) : est-ce une formulation humoristique une solution soufflée au metteur en scène pour une apparition surnaturelle, à savoir une projection d'image avec une voix off ou de l'humour? Avec Giraudoux, on ne sait jamais ${ }^{28}$. 


\section{L'humour}

dialogue entre le Président et Victor, le garçon de café de Cantique des cantiques, par une gestuelle caricaturale qui met en valeur la cocasserie de la comparaison :

La caissière, qui entre temps a gravi un haut comptoir avec les précautions d'un cocher d'omnibus. ( $C, 1$, p. 728).

L'humour permet de créer des effets comiques en mettant à distance le surnaturel tout en se moquant gentiment d'un personnage :

Auguste : tu vas rentrer, Ondine ! Je compte trois. Si à trois tu n'as pas obéi, je tire le verrou... Tu couches dehors. Coup de tonnerre.

Eugénie : Tu plaisantes!

Auguste : Tu vas voir si je plaisante !... Ondine, une ! Coup de tonnerre.

Eugénie : C'est assommant, ces coups de tonnerre à la fin de tes phrases!

[...]

Auguste : Ondine, deux! Coup de tonnerre.

Eugénie : Tu es insupportable!

Auguste : Ondine, trois! Pas de coup de tonnerre.

Eugénie, dans l'attente du coup de tonnerre : Finis, finis, mon pauvre Auguste!

Auguste : Moi, j'ai fini! (Il tire le verrou.). Voilà !... Nous voilà en paix pour le dîner.

La porte s'ouvre toute grande. Auguste et Eugénie se retournent au fracas. Un chevalier en armure est sur le seuil. (Ond., I, p. 763).

La nature - ou Ondine et le Roi des ondins - répond à Auguste par les manifestations de l'orage. La première didascalie "répond" avec un temps d'avance, si bien qu'à la troisième sommation d'Auguste, l'on en serait logiquement au... quatrième coup de tonnerre: la nature s'abstient et le facétieux auteur écrit donc: «Pas de coup de tonnerre. »! Auguste s'obstine à faire preuve d'autorité avec le verrou, mais à peine a-til exprimé sa satisfaction que sa précaution se révèle inutile. Le mot " fracas » suggère encore l'orage; or le surnaturel fait place à la brutalité soldatesque et au lieu de l'héroïne attendue, nous avons un chevalier errant.

61 L'humour noir n'est pas exclu: "Irma et le Sourd muet exeunt, comme dirait ce dernier.» (FC, II, p. 990). Outre l'emploi de la formule latine, le paradoxe fait sourire, et pourtant il est justifié préalablement par le jeu de «traduction» de la lettre circulaire dictée par la Folle au sourd muet - il faut être Giraudoux pour inventer un tel secrétaire -, « Irma traduisant », c'est-à-dire répétant ce que dicte la Folle et y ajoutant du latin de son cru (FC, I, p. 987).

Humour macabre: "On emmène Yami, ou on le tue sur place, selon l'humeur du metteur en scène. » (Jud., II, 2, p. 240). Double clin d'œil, l'un en relation avec la fable puisque Yami est le bourreau des Assyriens, l'autre en rapport avec les choix de Giraudoux qui rejette le pouvoir absolu du metteur en scène tel qu'il existe en Allemagne ${ }^{29}$.

Giraudoux établit donc tout un jeu avec les didascalies. Il cède peu aux conventions en matière de décor et de costumes et, s'il le fait, la place et la formulation des didascalies descriptives les éloigne aussi bien de l'actualisation que du pittoresque, à l'exception de la caricature ou de l'extravagance. Son sens du théâtre lui fait privilégier les didascalies narratives qui sont en prise directe sur l'action, le jeu, qui créent des effets comiques ou inquiétants. Son goût de la théâtralisation trouve dans l'intrication des répliques et des didascalies l'opportunité de jeux subtils. Enfin, nombre de didascalies paraissent 
peu prescriptives, et semblent davantage réservées à la lecture, à moins d'envisager d'autres manières de les traiter à la représentation.

Giraudoux, dans ce domaine comme dans bien d'autres, est inclassable, jouant du texte à lire et du texte à jouer, lui qui affirmait que «le vrai théâtre est dans les bibliothèques » alors qu'il venait aux répétitions et appelait Jouvet sa "deuxième Muse ».

\section{NOTES}

1. Nous excluons de notre réflexion les intitulés d'œuvres, les listes de personnages, les indications de découpage en actes, scènes, ainsi que les désignations des personnages locuteurs dans le dialogue.

2. A. Helbo, (dir.), Théâtre, modes d'approche, Klincksieck, « Méridiens », 1987, p. 173.

3. S. Golopentia, M. Martinez Thomas, Voir les didascalies, Toulouse le Mirail, Presses Universitaires du Mirail, CRIC, « Ibericas » $n^{\circ} 3$ \& OPHRYS, Paris, 1994, p. 12.

4. Dans une des versions primitives de Siegfried, une indication temporelle s'appuie sur un objet: «On allume les becs de gaz » (fr. 6, ms. 2, dactyl. 1 et 2) » (TC [Pl.], Notes et variantes, p. 1240). Dans aucune de ses pièces, Giraudoux n'utilise un tel moyen de préciser le moment de l'action.

5. J.-P. Ryngaert, Introduction à l'analyse du théâtre, Paris, Bordas, 1991, p. 24.

6. Encore faut-il parfois prendre avec précaution ces informations liminaires : pour Siegfried, J. Body note que «les indications concernant le décor, la sculpture et les costumes sont tirées du livre de bord de la Compagnie des Champs Elysées (fonds Jouvet) et qu'elles ne figurent dans aucune édition » (TC [Pl.] p.1239).

7. Temple dont on fermait les portes en temps de paix.

8. «Le balcon (avec un mur épais) ne doit pas être fermé - de manière à ce que le Spectre puisse entrer par là. Au moment de son entrée. Au moment de son entrée, les murs blancs vont devenir translucides, puis transparents et l'acteur longeant le décor aura l'air de marcher sur les toits gris et roses de la petite ville avant d'arriver sur le balcon [...].» (L. Jouvet, «Décoration d'Intermezzo ", Louis Jouvet, BN, 1961, p. 11-12), cité dans TC (Pl.), p. 147, n. 1 de p. 333.

9. . Op. cit., p. 83.

10. «Le double baisser de rideau est un effet de théâtre. », écrit J. Body (TC [Pl.], p. 1525).

11. M. Issacharoff, Le Spectacle du discours, Paris, Librairie José Corti, 1985, p. 39.

12. Contrairement au théâtre de Beaumarchais, mais en cela, Giraudoux ne diffère pas de ses contemporains.

13. « Il y a un réalisme dans la littérature fantaisiste et autrement difficile à saisir et à tenir que dans la littérature des naturalistes. » (Note copiée par C. Weil dans le cartonnier 10 des papiers Giraudoux conservés par son fils, note dont l'on ignore la date, citée par J. Body dans ORC, p. XXVIII).

14. En pleine querelle sur la séparation de l'Eglise et de l'Etat, si l'on reporte la jeunesse de Joséphine, comme celle de ses amies, à la Belle Epoque.

15. G. Wilson écrit à l'occasion de la reprise de la pièce : «Oui, cette Folle ne s'habille pas au rayon de confection d'un grand magasin : ce qu'elle revendique, par sa vêture extravagante, c'est le droit imprescriptible à la singularité, à la différence, à l'aventure, à la poésie, à la liberté. Car 
La Folle [sic] est aussi un admirable poème de combat pour la liberté. " ( L'Avant-Scène théâtre $\mathrm{n}^{\circ}$ 352,1966, p. 44).

16. Une autre didascalie descriptive, rapide croquis, se trouve à l'acte $\mathrm{I}$ : « Un petit vieux se faufile entre les tables, pommadé, ganté, pochette au vent. » (FC, II, p. 967).

17. Faut-il rappeler l'intérêt de Giraudoux pour le septième art, intérêt qui le conduisit à écrire des scénarios de films?

18. Sommes-nous si loin de l'idée qu'il doit être non un décor, mais une « machine à jouer ", idée défendue par les Constructivistes? Mais les dispositifs scéniques constructivistes se donnaient à lire comme tels, ne cherchant en aucune manière à figurer un décor, alors que nous savons que Jouvet, pour Electre, a mis en place derrière le décor des escaliers et des tambours qui permettaient aux acteurs d'apparaître soudainement aux yeux du public, en préservant l'illusion théâtrale.

19. Encore que cette dernière se comporte comme une femme ou une actrice lorsqu'elle "reparaît outrageusement fardée » (ibid.) pour faire oublier sa pâleur, c'est-à-dire combien elle est en cette fin d'acte I déjà fragile.

20. Cf. G. Teissier, "Apollon bifrons ou les équivoques visitations de Jean Giraudoux ", Présence de l'Antiquité, Colloque de Tours, 1994, repris dans Des Mots et des mondes, p. 157-177 et le CJG nº 36 consacré à cette pièce.

21. Voici l'exemple d'une didascalie qui semble réservée à la lecture, or elle est indirectement prescriptive, invitant le metteur en scène à trouver le moyen de signifier cette ambivalence du personnage, ce qu'avait fait Jouvet : pour la création, le décorateur Tchélitchew lui avait attribué un accessoire bivalent, faux et quenouille à la fois et « un tablier maculé [qui] montrait en anamorphose une tête de mort.» (cf. G. Teissier ; "Les attributs de la fille de vaisselle, même perçus de manière contradictoire, en font explicitement une figure de la Parque ou de la mort.» Notes d'Ondine, TC [P]., p. 1229).

22. Sa précédente sortie après l'aveu de Lucile mêlait le tragique et le bouffon : "Il part avec ses pistolets, bousculant le greffier qui entre. » (Luc., III, 2, p. 1099).

23. T. Kowzan, «Vers la surthéâtralisation dans l'œuvre dramatique de Jean Giraudoux », Paris, Grasset, CJG n¹2, 1983, p. 100-112.

24. «La scène représente la terrasse d'une villa d'où l'on voit dans le lointain deux villes: Sodome et Gomorrhe. Le jardinier travaille à un massif. Dans un nimbe d'or et de feu, l'archange apparaît. » (Sod., "Prélude », TC [Pl.], p. 1691). Dans la version définitive, Giraudoux a fait le choix de la sobriété, reportant la mention de la villa au second acte, en situation, ce qui a pour conséquence la modification du statut de l'espace scénique et de sa perception par le lecteur. Il rejoint ainsi l'idéal du "plateau nu » de Copeau. En outre, l'absence de didascalie prescriptive laisse désormais toute liberté au metteur en scène, Giraudoux faisant entièrement confiance à Jouvet.

25. L'attention portée à la lumière par Jouvet est connue, nous la retrouvons dans L'Impromptu de Paris : le régisseur essaie les « casseroles » sur le député mais tout passe par le dialogue (IP, 3, p. 698-699).

26. C. Hallak, « La dialectique du vrai et du faux », CJG $n^{\circ} 25$, p. 52.

27. Cf. FC, I, p. 955.

28. Contrairement à Claudel qui, dans les didascalies, précise l'emploi de projetions, d'écran (voir Le Soulier de satin).

29. Cf. "Le théâtre contemporain en France et en Allemagne», repris dans Littérature, Paris, Grasset, 1941. 